\title{
Vital Pulp Therapy Challenges and Promises in Permanent Teeth
}

ISSN: 2637-7764

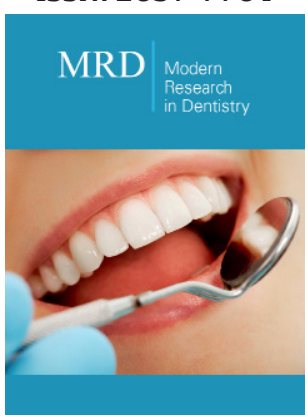

${ }^{* 1}$ Corresponding author: Fahda Algahtani, Riyadh, Princess Nourah Bint Abdulrahman University, Saudi Arabia

Submission: 阽 February 27, 2019

Published: 睡 March 05, 2019

Volume 3 - Issue 4

How to cite this article: Fahda A. Vital Pulp Therapy Challenges and Promises in Permanent Teeth. Mod Res Dent. 3(4). MRD.000569.2019.

DOI: 10.31031/MRD.2019.03.000569

Copyright@ Fahda Algahtani, This article is distributed under the terms of the Creative Commons Attribution 4.0 International License, which permits unrestricted use and redistribution provided that the original author and source are credited.

\section{Fahdla Algahtani*}

Clinical dentistry department, Princess Nourah Bint Abdulrahman University, Saudi Arabia

\begin{abstract}
The question about the exact indications and contraindications of vital pulp therapy in permanent teeth never settled down. Because the answer involves multiple factors such as understanding the complex nature of the carious disease, knowing the pathological state inside the pulp, and recognizing the capabilities and the limits of the biomaterials in stimulating repair. This review examines some of the challenges and promises of vital pulp therapy for permanent teeth.
\end{abstract}

Keywords: Vital pulp therapy; Pulp capping; Restoration

Abbreviations: RCT: Root Canal Treatment; MTA: Mineral Trioxide Aggregate; VPT: Vital Pulp Therapy

\section{Introduction}

Dental caries is one of the most prevalent oral diseases in many parts of the world [1] The primary accepted treatment for this pathology is excision of dentinal infected tissue and restorative replacement with suitable material. Although caries targets the dental hard tissue, the attack will impact the pulp [2] The dentin-pulp complex acts as a vital organ that responds to the bacterial attack with inflammation [2,3] The dental inflammation is unique because of the presence of highly specialized cells, the odontoblasts that extend variably within dentinal tubules into the dentin matrix through the odontoblastic processes [2,3] Also, the presence of the blood vessels and nerves within narrow hard vicinity in the absence of adequate lymphatic supply [2] The inflammation cascade can proceed to cause irreversible damage and pulp necrosis in absence of intervention for the intense carious attack $[2,3]$ The pulp involvement in the clinical procedure complicates the restorative treatment plan and increases the emotional and financial cost for the patient. Vital pulp therapy "VPT" is a palliative treatment that meant to increase the life span of the vital tooth and delay the need for intensive treatment and further loss of dentinal tooth structure. However, this option is frequently challenged and the clear indications are occasionally ambiguous. This article summarizes vital pulp therapy challenges in the past and the possibilities in the near future.

\section{Vital pulp therapy challenges}

Distinguishing the concept of regeneration from repair in the wound healing of the pulp-dentin complex: Regeneration and repair are two different types of wound healing $[4,5]$ The regeneration involves the recruitment of identical producer cells to synthesize and replace the lost tooth structure and restore the original biological function [5] On the other hand, repair involves closing the defect with a different tissue produced by a different cell [5] Unlike the regeneration, the biological function of the repaired tissue is not attained or imperfect. The differentiation of stem cells into odontoblasts happens twice separately before primary dentin formation of the deciduous and the permanent teeth [5,6] The odontoblastic process allows hydroxyapatite crystals to mineralize the surrounding dentin matrix during dentinogenetic [6] The formation of secondary dentin throughout life is a physiological protective process $[3,5]$ The tertiary dentin is then secreted in response to adverse event or stimuli such as dental caries which can be reactionary or reparative depending on the type of cell employed $[2,3]$ The reactionary dentin that was formed by the original odontoblasts 
is considered as another physiological defense mechanism [2,3] However, reparative dentin is secreted by odontoblast-like cells that are newly differentiated from pulp mesenchymal cells and different from the original odontoblast in structure [2,3,5] The newly differentiated odontoblast-like cells don't have odontoblastic process extending within the original dentinal tubules rather than being able to build new dentin that is different from the original in the structure, the direction of dentinal tubules, and the composition $[2,3,5]$ The reparative dentin formation, therefore, is considered as wound repair [5] In conclusion, wound repair rather than regeneration is the present clinically achievable goal for vital pulp therapy in permanent teeth.

The healing ability and repair capacity of the aging pulp: The odontoblast flattens with age and the pulp space becomes smaller due to secondary and tertiary dentin deposition [7] The fibroblast and collagen matrix will occupy the majority of space inside the pulp cavity [7] Therefore, obtaining a thermal response in vitality tests is more challenging. This could adversely influence the pulp response to new treatment. Especially if the tooth survived a history of active cavitation's, multiple restorations, arrested caries lesions, attrition, malocclusion, etc. The histological condition of the normal pulp in restored teeth was similar to reversible pulpitis [8] The blood vessels are widened and the inflammatory cells are high in number [8] The normal histological condition was found only in teeth that have no history of cavitation or restoration [8] However, vital pulp therapy is still possible in aged teeth because all cases are different in terms of the history, the new defect location, pulp diagnosis, calcifications, restorative treatment planning, and patient wishes.

The clinical diagnosis of the degree of pulp inflammation in asymptomatic cases: The histological pictures in $84 \%$ of symptomatic pulpitis cases are composed of an abscessed zone close to the deepest carious defect followed by heavy infiltration of inflammatory cells [8] The pulp is judged in these cases as histologically irreversible consistently in the majority of cases [8] However, this is not an absolute contraindication of vital pulp therapy since it only signifies the presence of structural damage. The presence of the abscessed area may necessitate partial pulpotomy or complete pulpotomy for the least to exclude the area of necrosis and the heavy infiltration of inflammatory cells. The degree of hemostasis achieved in washing the open wound with $6 \% \mathrm{NaOCl}$ up to ten minutes hints the health state of the remaining exposed pulp [9] If the hemostasis was achieved then remaining tissue is judged as healthy [9]. The histological picture in asymptomatic cases can vary between normal, reversible and irreversible inflamed conditions [8,10] The American Association of Endodontists AAE classifies the cases with deep caries and normal vitality response as asymptomatic irreversible pulpitis [11] The depth of caries was emphasized in the classification to be closer to the classical histological findings in these cases. The final diagnosis in asymptomatic cases is made after removing caries. If pulp was exposed then the degree of hemostasis hints the health of the exposed tissue and aid in deciding on either removing the inflamed part or the entire pulp [9] The reliability of any treatment depends on the understanding of all factors relative to prognosis. Also, the aseptic environment and precise clinical technique are essential in dealing with frank pulp exposure [12]

Remaining tooth structure and the restorative treatment plan: Sometimes elective root canal treatment "RCT" is considered due to restorative reasons. For example, the tooth has a few remaining walls which mandate restoring with a post and core. Another example is when overdenture or root amputation is planned [13] Moreover, the full treatment plan might indicate the extraction of vital teeth for the interest of making an implant supported denture. Therefore, it's always important to start with a restorative plan ahead to set a direction for the treatment.

The positive outcome of dentin bridge formation versus pulp space obliteration: It's clinically relevant to find partial to complete pulp space obliteration in teeth which had a history of vital pulp therapy in young dentition. Although thick dentin bridge is a sign of treatment success in pulpotomy cases, accessing these canals for RCT can be challenging. However, a recent prospective randomized clinical trial confirmed the absence of complete pulp obliteration after three years of follow up in permanent teeth [14] Also, the dentin bridge was not evident radiographically for the majority of cases [14]. The quality of the dentin bridge was described as being porous with some calcification $[15,16]$ The potential challenge of accessing root canals in these cases is yet to be addressed in future clinical studies.

The critique for clinical studies addressing vital pulp therapy: The clinical studies for vital pulp therapy were criticized for being inconclusive regarding the factors that influence the success of the treatment [17] Partly due to the absence of full gathered information in relation to patient age, symptoms, and depth of caries [17] Some clinical studies lack information regarding the calibration or randomization of evaluators or procedures which increase the risk of bias [18] The depth of caries and the nature of the carious activity are important parameters in understanding the extent and reversibility of inflammation [19] Because the success potential of the treatment can change in each different situation. Therefore, recalling pathological pulp exposure alone was clinically indistinctive and provide incomplete information for the reviewer [19]

Modern root canal therapy: The modern RCT is moving toward accomplishing the goals of endodontic treatment with minimal removal of the tooth structure using advanced technology, instruments, and materials. The conventional endodontic access is not needed anymore to visualize the canals in the presence of a microscope [20] The cone beam scan is not an invasive way to explore rare anatomies, additional canals, and explore mishaps [20] The ultrasonic tip allows visualization and accuracy in movement. The rotary files and technologies of activated irrigation simplified the cleaning and shaping [20] The matching cones and ceramic sealers allow for pressure less single cone obturation. These advantages might influence the clinician and direct him away from exploring the VPT option in borderline cases for the benefit of the predictable and fair alternative RCT option. 


\section{The promises in vital pulp therapy}

The availability of bioceramic materials: Mineral trioxide aggregate "MTA" has been studied extensively in the literature [21-23] The material is biocompatible, bioactive, antibacterial, and provide good sealing ability [21,22] The MTA disadvantages were discoloration and long setting time [23] The subsequent MTA bioceramic derivatives proclaim improvements in the material manipulation, setting time, and discoloration while preserving the main composition [24] The clinical success of vital pulp therapy using bio ceramic materials was high $[9,17,23]$.

Introducing molecular technique to diagnose the pulp histological status: The contemporary deep understanding of inflammation cycle in the pulp may promote the accuracy of the clinical diagnosis. Information about the concentration and type of inflammatory mediator collected directly from the tooth can indicate the type of inflammation $[25,26]$ The promise is to develop a chairside tool that can collect and analyze a sample of the dental fluid or a bleeding point in the exposed pulp to give the actual diagnosis $[25,26]$ The primary boundary is knowing the exact concentration of inflammatory mediators in irreversible inflammation and not only the type of mediator [25] The analysis of gingival crevicular fluid is an atraumatic way of analyzing pulp inflammation and is clinically applicable [26] However, the drawback of using this technique is identifying mediators that are unique for the pulp and independent from the periodontal ligament [26]

Achieving hemostasis appears to be a feasible method in diagnosing the condition of exposed pulp or remaining pulp: Sodium hypochlorite was used in the concentration of $6 \%$ for ten minutes primarily for achieving hemostasis [9] The persistent bleeding was a sign of irreversible inflammation and further excision of the exposed pulp is required to expose the healthy section [9] However, ten minutes might be too slow for a busy dentist who might be impatient and lose hope after one minute of profuse bleeding.

The positive outcomes of the incomplete removal of carious dentin in clinical studies: The caries cavitation in the enamel layer is a reversible condition and remineralization often occurs $[27,28]$ On the other hand, dentin cavitation was thought to be an irreversible condition and surgical removal of the pathology and destructed structure was reasonable [29] The research is constantly challenging this treatment philosophy and partial caries excavation is proving to be a better alternative to complete caries excavation procedure [29] The injury of the odontoblast and predentin after surgical removal of a carious lesion can be more dramatic than a cavitation insult [30] Moreover, the reversibility of dentin cavitation by the mineralized dentinal fluid is possible when complete isolation of bacteria from the external oral environment was achieved [29,31] One cohort study and a more recent systematic analysis concluded that complete caries removal is not necessary for successful management of dental caries providing that the margins of the tooth are cleaned and the restoration is well adapted [31-33] More detailed clinical studies that describe the amount and quality of caries left behind was encouraged [32] Since the composite bonding quality to carious dentin is questionable, then glass ionomer can be used as a base below the composite or as a temporary restoration in stepwise excavation procedure [29]

The interest in developing new pulp capping materials: Growth factors that promote hard tissue formation and dentinogenesis are potential future candidates for pulp capping $[16,34]$ The destructive inflammatory cycle can be also regulated toward tissue repair using anti-inflammatory peptides [16]

\section{Conclusion}

The discipline in VPT is continuously evolving and has a positive stand. The current understanding of caries and remineralization has shifted the treatment philosophy to more conservative surgical management of deep carious lesions. The pulp inflammatory mediators are known and a chairside diagnostic tool that accurately detects pulp state is possible. The clinical studies are reporting positive outcome using the present techniques and materials.

\section{Conflict of interest}

The author has no conflict of interest relative to the content provided in this review.

\section{References}

1. Frencken J, Sharma P, Stenhouse L, Green D, Laverty D, et al. (2017) Global epidemiology of dental caries and severe periodontitis-A comprehensive review. J Clin Periodontol 44(Suppl 18): S94-S105.

2. Bjørndal L, Mjör I (2001) Pulp-dentin biology in restorative dentistry. Part 4: Dental caries- characteristics of lesions and pulpal reactions. Quintessence Int 32(9): 717-736.

3. Smith A (2002) Pulpal responses to caries and dental repair. Caries Res 36(4): 223-232.

4. Lin L, Huang G (2015) Regeneration and repair in endodontics- A special issue of the dentistry journal. Dent J (Basel) 3(3): 77-78.

5. Lin L, Rosenberg P (2011) Repair and regeneration in endodontics. Int Endod J 44(10): 889-906.

6. Ruch J, Lesot H, Begue KC (1995) Odontoblast differentiation. Int J Dev Biol 39(1): 51-68.

7. Bernick S, Nedelman C (1975) Effect of aging on the human pulp. J Endod 1(3): 88-94.

8. Ricucci D, Loghin S, Siqueira JF (2014) Correlation between clinical and histologic pulp diagnoses. J Endod 40(12): 1932-1939.

9. Witherspoon D (2008) Vital pulp therapy with new materials: New directions and treatment perspectives-permanent teeth. J Endod 34(7Suppl): S25-S28.

10. Baume L (1970) Diagnosis of diseases of the pulp. Oral Surg, Oral Med, Oral Pathol 29(1): 102-116.

11. Glickman G, Schweitzer J (2003) Endodontic diagnosis, in ENDODONTICS: Colleagues for Excellence. American Assoc of Endodontists.

12. Mjör I (2002) Pulp-dentin biology in restorative dentistry. Part 7: The exposed pulp. Quintessence Int 33(2): 113-135.

13. Mohamed H, Ahmed A (2014) Elective root canal treatment: A review and clinical update. Endo (Lond Engl) 8(2): 139-144. 
14. Awawdeh L, Al Qudah A, Hamouri H, Chakra RJ (2018) Outcomes of vital pulp therapy using mineral trioxide aggregate or biodentine: A prospective randomized clinical trial. J Endod 44(11): 1603-1609.

15. Reston EG, de Souza Costa CA (2009) Scanning electron microscopy evaluation of the hard tissue barrier after pulp capping with calcium hydroxide, mineral trioxide aggregate (MTA) or ProRoot MTA. Aust Endod J 35(2): 78-84.

16. Morotomi T, Washio A, Kitamura C (2019) Current and future options for dental pulp therapy. Jpn Dent Sci Rev 55(1): 5-11.

17. Aguilar P, Linsuwanont P (2011) Vital pulp therapy in vital permanent teeth with curiously exposed pulp: A systematic review. J Endod 37(5): 581-587.

18. (2009) Evidenced-based review of clinical studies on indirect pulp capping. J Endod 35(8): 1147-1151.

19. Bjørndal L, Demant S, Dabelsteen S (2014) Depth and activity of carious lesions as indicators for the regenerative potential of dental pulp after intervention. J Endod 40(4 Suppl): S76-S81.

20.Gluskin AH (2014) The standard of practice in contemporary endodontics, in ENDODONTICS: Colleagues for excellence. American Assoc of Endodontists and the AAE Foundation.

21. Parirokh M, Torabinejad M (2010) Mineral trioxide aggregate: A comprehensive literature review-- Part I: chemical, physical, and antibacterial properties. J Endod 36(1): 16-27.

22. Torabinejad M, Parirokh M (2010) Mineral trioxide aggregate: A comprehensive literature review-- part II: Leakage and biocompatibility investigations. J Endod 36(2): 190-202.

23. Parirokh M, Torabinejad M (2010) Mineral trioxide aggregate: A comprehensive literature review-- Part III: Clinical applications, drawbacks, and mechanism of action. J Endod 36(3): 400-413.
24.Wang Z (2015) Bioceramic materials in endodontics. Endod Topics $32(1): 3-30$

25. Zanini M, Meyer E, Simon S (2017) Pulp inflammation diagnosis from clinical to inflammatory mediators: A systematic review. J Endod 43(7): 1033-1051.

26. Rechenberg DK, Galicia JC, Peters OA (2016) Biological markers for pulpal inflammation: A systematic review. PloS one 11(11): e0167289.

27. Diefenderfer K, Stahl J (2008) Caries remineralization therapy: Implications for dental readiness. Mil Med 173(1 Suppl): 48-50.

28. Ten Cate J, Fejerskov O (2003) Chemical interactions between the tooth and oral fluids. In: Fejerskov 0, Kidd E (Eds.), Dental caries: The disease and its clinical management. (2nd edn), Blackwell Publishing, Oxford, pp. 49-69.

29. Bjørndal L (2002) Dentin caries: Progression and clinical management. Oper Dent 27(3): 211-217.

30. Mjör I (2001) Pulp-dentin biology in restorative dentistry. Part 2: Initial reactions to preparation of teeth for restorative procedures. Quintessence Int 32(7): 537-551.

31. Thompson V, Craig RG, Curro FA, Green WS, Ship JA (2008) Treatment of deep carious lesions by complete excavation or partial removal: a critical review. J Am Dent Assoc 139(6): 705-712.

32. Schwendicke F, Dörfer C, Paris S (2013) Incomplete caries removal: A systematic review and meta- analysis. J Dent Res 92(4): 306-314.

33. RickettsD, Kidd E, Innes N, Clarkson J (2006) Complete or ultraconservative removal of decayed tissue in unfilled teeth. Cochrane Database Syst Rev (3): CD003808.

34. Rutherford B, Fitzgerald M (1995) A new biological approach to vital pulp therapy. Crit Rev Oral Biol Med 6(3): 218-229.

For possible submissions Click below: 